C. Rotthaus

Nagoya Math. J.

Vol. 74 (1979), 123-135

\title{
UNIVERSELL JAPANISCHE RINGE MIT NICHT OFFENEM REGULÄREM ORT
}

\author{
CHRISTEL ROTTHAUS
}

Im Zusammenhang mit dem von Grothendieck in [2] Chap. IV (7.4.8) gestellten Problem "Ist die ideal-adische Komplettierung eines ausgezeichneten Ringes wieder ausgezeichnet?" scheint aufgrund der Arbeiten von Marot [3] und Valabrega [8] im lokalen Fall die folgende Frage interessant zu sein: " $R$ sei ein lokaler universell japanischer Ring. Ist der singuläre Ort jeder endlich erzeugten $R$-Algebra $A$ abgeschlossen in Spec $(A)$ ?" Diese Frage wird hier negativ beantwortet, d.h. wir werden einen lokalen universell japanischen Ring $A$ konstruieren, dessen singulärer Ort nicht abgeschlossen in der Zariski-Topologie von $\operatorname{Spec}(A)$ ist.

Die Bezeichnungen sind dieselben wie in [2]. Unter der Komplettierung $\hat{A}$ eines lokalen Ringes $A$ mit maximalem Ideal $m_{A}$ verstehen wir immer die Komplettierung von $A$ nach der $m_{A^{-}}$adischen Topologie.

Zunächst sei an einige Beziehungen über universell japanische Ringe erinnert:

Theorem 1. A sei ein lokaler noetherscher Ring. Dann sind folgende Aussagen äquivalent:

(i) A ist universell japanisch.

(ii) Die formellen Fasern von $A$ sind geometrisch reduziert.

(iii) Für jeden nullteilerfreien Restklassenring $B$ von $A$ (d.h. $B=A / \mathfrak{p}$ für ein $\mathfrak{p} \in \operatorname{Spec}(A))$ mit Quotientenkörper $K=Q(B)$ sind die folgenden beiden Bedingungen erfüllt:

( $\alpha$ ) $\hat{B}$ ist reduziert

( $\beta$ ) Für alle minimalen Primideale $\mathfrak{B} \in \operatorname{Spec}(\hat{B})$ ist die Körpererweiterung $\hat{B}_{\mathfrak{B}} / K$ separabel.

Beweis. [2] Chap. IV (7.7.2) und (7.6.4)

Received July 14, 1978.

Revised August 4, 1978. 
Lemma. A sei ein lokaler universell japanischer Ring; $\mathfrak{p} \in \operatorname{Spec}(A)$ und $\mathfrak{\beta} \in \operatorname{Spec}(\hat{A})$ minimal mit $\mathfrak{P} \cap A=\mathfrak{p}$. Dann folgt:

$$
\mathfrak{p} \in \operatorname{Reg}(A) \leftrightarrow \mathfrak{P} \in \operatorname{Reg}(\hat{A})
$$

Beweis. $\quad$ Da $\mathfrak{p} \hat{A}$ reduziert ist, folgt $\mathfrak{p} \hat{A}_{\mathfrak{P}}=\mathfrak{P} \hat{A}_{\mathfrak{\beta}} . \quad$ Aus $\operatorname{dim} A_{\mathfrak{p}}=\operatorname{dim} \hat{A}_{\mathfrak{\beta}}$ folgt dann die Behauptung.

\section{§1. Vorbereitungen}

$\boldsymbol{Q}$ sei der Körper der rationalen Zahlen; $A_{i}, B_{j}, i, j \in N$, seien Unbestimmte über $\boldsymbol{Q}$ mit $A_{i} \neq A_{j}$ und $B_{i} \neq B_{j}$ für alle $i, j \in N$ mit $i \neq j$ und $A_{i} \neq B_{j}$ für alle $i, j \in N$. Dann ist $K=Q\left(A_{i}, B_{j}\right)_{i, j \in N}$ ein Körper mit abzählbar unendlich vielen Elementen. $X, Y, Z, T$ seien Unbestimmte über $K$. Der Polynomring $K[X, Y, Z, T]_{(X, Y, Z, T)}$ enthält wieder abzählbar unendlich viele Elemente; insbesondere ist die Menge der Primelemente in $K[X, Y, Z, T]_{(X, Y, Z, T)}$ abzählbar. Wir wählen nun eine Teilmenge $\mathscr{P}$ $\subseteq Q\left[A_{i}, B_{j}\right]_{i, j \in N}[X, Y, Z, T]$ mit folgenden Eigenschaften:

(1) Die Elemente aus $\mathscr{P}$ sind Primelemente in $K[X, Y, Z, T]_{(X, Y, Z, T)}$ und irgend zwei Elemente aus $\mathscr{P}$ sind nicht assoziiert in $K[X, Y, Z$, $T]_{(X, Y, Z, T)}$.

(2) $X \in \mathscr{P}$ und $X+Y^{k} \in \mathscr{P}$ für alle $k \in N$.

(3) Ist $P$ ein Primelement in $K[X, Y, Z, T]_{(X, Y, Z, T)}$, so gibt es ein zu $P$ assoziiertes Primelement $P^{\prime} \in \mathscr{P}$.

Sei $\mathscr{P}=\left\{p_{n}\right\}_{n \in N}$ eine Abzählung von $\mathscr{P}$ mit $p_{1}=X$. Wir setzen:

$$
q_{n}=\prod_{i=1}^{n} p_{i}
$$

und definieren für Elemente

$$
F=\sum_{0 \leqq i, j, k, \ell \leqq n} a_{i j k \ell} X^{i} Y^{j} Z^{k} T^{\ell} \in K[X, Y, Z, T]
$$

mit $a_{i j k \ell} \in K$ :

$$
\operatorname{Grad} F=\partial F=\operatorname{Max}\left\{i+j+k+\ell \mid a_{i j k \ell} \neq 0\right\} .
$$

Mit $t_{n}:=\partial q_{n}$

$$
r_{n}:=\left[\left(t_{n}+1\right) !\right]^{2}
$$

bilden wir nun die folgenden Elemente aus dem formalen Potenzreihenring: 


$$
\begin{aligned}
& \tau:=\sum_{i=1}^{\infty} A_{i} q_{i}^{r_{i}} \\
& \sigma:=\sum_{i=1}^{\infty} B_{i} q_{i}^{r_{i}}
\end{aligned}
$$

Im folgenden benötigen wir, daß das Element $\omega_{0}=(Z+\tau)(T+\sigma) \in K[[X$, $Y, Z]$ ] algebraisch unabhängig über $K(X, Y, Z, T)$ ist. Dazu zeigen wir:

SATz 1. Die Menge $\{\sigma, \tau\}$ ist algebraisch unabhängig über $K(X, Y, Z, T)$.

Beweis. Andernfalls gibt es ein $F\left(S_{1}, S_{2}\right) \in K[X, Y, Z, T]\left[S_{1}, S_{2}\right] \backslash\{0\}$ mit $F(\sigma, \tau)=0$, etwa:

$$
F=\sum_{0 \leq i, j \leq q} a_{i j} S_{1}^{i} S_{2}^{j}
$$

mit $a_{i j} \in K[X, Y, Z, T]$. Sei $d:=\operatorname{Max}\left\{\partial a_{i j} \mid 0 \leqq i, j \leqq q\right\}$. Dann läßt sich nach Multiplikation vom $F$ mit einem Element $\neq 0$ aus $K$ ein $r \in N$ finden mit $r>\operatorname{Max}\{d, 2 q\}$, so daß

$$
a_{i j} \in Q\left[A_{\nu}, B_{\mu}\right]_{1 \leqq \nu, \mu \leqq r}[X, Y, Z, T] .
$$

Im folgenden setzen wir $M_{n}=Q\left[A_{\nu}, B_{\mu}\right]_{1 \leqq \nu, \mu \leqq n}[X, Y, Z, T]$ für alle $n \in N$. Dann gibt es ein $s \geqq r$ mit $q_{i} \in M_{s}$ für $1 \leqq i \leqq r$. Wir unterscheiden nun drei Fälle:

1. Fall. $q_{1}, \cdots, q_{t} \in M_{s}, q_{t+1} \notin M_{s}$ für ein $t$ mit $s>t \geqq r$.

Setze $m:=t ; p:=s$.

2. Fall. $q_{1}, \cdots, q_{s}, q_{s+1} \in M_{s}$.

Setze $m:=s ; p:=s$.

3. Fall. $q_{1}, \cdots, q_{s} \in M_{s}, q_{s+1} \notin M_{s}$.

Dann gibt es ein $h \geqq s$ mit

3.1: $q_{1}, \cdots, q_{h+1} \in M_{h}$ oder

3.2: $q_{1}, \cdots, q_{h} \in M_{h}, q_{h+1} \notin M_{h+1}$

denn $\mathscr{P}$ enthält unendlich viele Primelemente der Form $X+Y^{k} ; k \in N$. In den Fällen 3.1 und 3.2 setze $\operatorname{man} m:=h$ und $p:=h$. Sei nun:

$$
\begin{aligned}
& P_{1}(X, Y, Z, T)=\sum_{\mu=1}^{m} B_{\mu} q_{\mu}^{r_{\mu}} \\
& P_{2}(X, Y, Z, T)=\sum_{\nu=1}^{m} A_{\nu} q_{\nu}^{r_{\nu}}
\end{aligned}
$$

Dann ist:

$$
P_{1}, P_{2} \in M_{p}
$$


Weiter definieren wir:

$$
G\left(S_{1}, S_{2}\right)=F\left(S_{1}+P_{1}, S_{2}+P_{2}\right)=\sum_{0 \leqq i, j \leqq q} b_{i j} S_{1}^{i} S_{2}^{j}
$$

wobei $b_{i j} \in M_{p}$ für alle $i, j \in N_{0}$ mit $0 \leqq i, j \leqq q$. Mit $F\left(S_{1}, S_{2}\right) \neq 0$ ist auch $G\left(S_{1}, S_{2}\right) \neq 0$, und es gilt

$$
G\left(\sigma-P_{1}, \tau-P_{2}\right)=F(\sigma, \tau)=0 .
$$

Weiter folgt für alle $i, j \in N_{0}$ mit $0 \leqq i, j \leqq q$ :

$$
\begin{aligned}
\partial b_{i j} & \leqq d+2 q r_{m} t_{m}=d+2 q\left[\left(t_{m}+1\right) !\right]^{2} t_{m} \\
& <\left[\left(t_{m+1}+1\right) !\right]^{2}=r_{m+1}
\end{aligned}
$$

Für $i>0$ oder $j>0$ gilt:

$$
\begin{aligned}
& \left(\sum_{n=m+1}^{\infty} B_{n} q_{n}^{r_{n}}\right)^{i}\left(\sum_{n=m+1}^{\infty} A_{n} q_{n}^{r_{n}}\right)^{j} \\
& =B_{m+1}^{i} A_{m+1}^{j} q_{m+1}^{(i+k) r_{m+1}}+\Delta_{i j}
\end{aligned}
$$

dabei ist $\Delta_{i j}$ eine Potenzreihe in $K[[X, Y, Z, T]]$, in der nur Terme vom Grad $\geqq r_{m+2}=\left[\left(t_{m+2}+1\right) !\right]^{2}$ vorkommen. Damit folgt:

$$
\begin{aligned}
G\left(\sigma-P_{1}, \tau-P_{2}\right)= & b_{00}+\sum_{\substack{0 \leq i, j \leq q \\
i \neq 0 \vee j \neq 0}} b_{i j} B_{m+1}^{i} A_{m+1}^{j} q_{m+1}^{(i+j) r_{m+1}} \\
& +\sum_{\substack{0 \leq i, j \leq q \\
0 \neq i \text { j\j0 }}} b_{i j} \Delta_{i j}=0
\end{aligned}
$$

Abschätzen der Grade der einzelnen Terme in (*) liefert:

(a) $\partial b_{00} \leqq r_{m+1}<r_{m+2}$

(b) $\quad \partial \sum_{\substack{0 \leq i, j \leq q \\ i \neq 0 \vee j \neq 0}} b_{i j} B_{m+1}^{i} A_{m+1}^{j} q_{m+1}^{(i+j) r_{m+1}}<r_{m+2}$

(c) $\sum_{\substack{0 \leq i, j \leq q \\ i \neq 0 \vee j \neq 0}} b_{i j} \Delta_{i j}=0$ oder der Grad der Monome in der Potenzreihe

$$
\sum_{\substack{0 \leq i, j \leq q \\ i \neq 0 \vee j \neq 0}} b_{i j} \Delta_{i j} \text { ist } \geqq r_{m+2}
$$

Gradvergleich liefert daraus:

$$
b_{00}+\sum_{\substack{0 \leq i, j \leq q \\ i \neq 0 \vee j \neq 0}} b_{i j} B_{m+1}^{i} A_{m+1}^{j} q_{m+1}^{(i+j) r_{m+1}}=0
$$

Nun folgt im

1. Fall. $A_{m+1}, B_{m+1}, b_{i j} \in M_{p}$ und

$$
q_{m+1} \notin M_{p} \text {. }
$$

Also ist $q_{m+1}$ algebraisch abhängig über $M_{p}$. Andererseits ist $a_{i j}=b_{i j}$ 
für $i+j=k_{0}$ mit $k_{0}=\operatorname{Max}\left\{i+j \mid a_{i j} \neq 0\right\} . \quad$ Da $a_{i j} \in M_{r}$ und da $\left\{A_{m+1}, B_{m+1}\right\}$ algebraisch unabhängig über $M_{r}$ ist, erhalten wir $\sum_{i+j=k_{0}} b_{i j} B_{m+1}^{i} A_{m+1}^{j} \neq 0$. Widerspruch.
2. Fall.$$
b_{i j}, q_{m+1} \notin M_{p}
$$

$$
A_{m+1}, B_{m+1} \notin Q\left[A_{\nu}, B_{\mu}\right]_{1 \leqq \nu, \mu \leqq p}
$$

Dann ist $\left\{A_{m+1}, B_{m+1}\right\}$ algebraisch abhängig über $M_{m}$. Widerspruch.

3. Fall. $3.1 b_{i j}, q_{m+1} \in M_{m}$.

Wie oben ergibt sich der Widerspruch: $\left\{A_{m+1}, B_{m+1}\right\}$ ist algebraisch abhängig über $M_{m}$. Im Fall $3.2 b_{i j}, A_{m+1}, B_{m+1} \in M_{m+1} q_{m+1} \notin M_{m+1}$ folgt wieder, daß $\left\{A_{r}, B_{r}\right\}_{r>m+1}$ algebraisch abhängig über $M_{m+1}$ ist. Widerspruch.

Damit folgt: $b_{i j}=0$ für alle $i, j ; 0 \leqslant i, j \leqslant q$; entgegen $G\left(S_{1}, S_{2}\right) \neq 0$. Im folgenden sei $\mathscr{P} \subseteq Q\left[A_{\nu}, B_{\mu}\right]_{\nu, \mu \in N}[X, Y, Z, T]$ eine Menge von Primelementen in $K[X, Y, Z, T]_{(X, Y, Z, T)}$ mit den Eigenschaften (1), (2) und (3). $\varphi: N \rightarrow \mathscr{P}$ sei eine Abzählung von $\mathscr{P}$ mit $\varphi(1)=X$. Dann definieren wir:

$$
\begin{aligned}
q_{\varphi_{n}}: & =\prod_{i=1}^{n} \varphi(i) \\
t_{\varphi_{n}}: & =\partial q_{\varphi_{n}} \\
r_{\varphi_{n}}: & =\left[\left(t_{\varphi_{n}}+1\right) !\right]^{2} \\
g_{\varphi_{n}}: & =Z+\sum_{i=1}^{n} A_{i} q_{\varphi_{i}}^{r_{\varphi_{i}}} \\
h_{\varphi_{n}}: & =T+\sum_{i=1}^{n} B_{i} q_{\varphi_{i}}^{r_{\varphi_{i}}}
\end{aligned}
$$

Das von $g_{\varphi_{n}}$ und $h_{\varphi_{n}}$ erzeugte Ideal $\mathfrak{p}_{\varphi_{n}}$ ist ein Primideal in $K[X, Y, Z$, $T]_{(X, Y, Z, T)}$, da $g_{\varphi_{n}}, h_{\varphi_{n}}, X, Y$ das maximale Ideal in $K[X, Y, Z, T]_{(X, Y, Z, T)}$ erzeugen. Für ein $r>n$ sei $\mathfrak{p}_{\varphi_{n}, r}$ das von

$$
\begin{aligned}
& g_{\varphi_{n}, r}:=g_{\varphi_{n}}+A_{n+1} q_{\varphi_{n}, r}^{r_{\varphi_{n}, r}} \\
& h_{\varphi_{n}, r}:=h_{\varphi_{n}}+B_{n+1} q_{\varphi_{n}, r}^{\varphi_{n}, r}
\end{aligned}
$$

erzeugte Primideal, dabei ist $q_{\varphi_{n}, r}:=q_{\varphi_{n}} \cdot \varphi(r)$ und $r_{\varphi_{n}, r}=\left[\left(t_{\varphi_{n}, r}+1\right) !\right]^{2}$ mit $t_{\varphi_{n}, r}=\partial q_{\varphi_{n}, r}$. Ist $s>r$, so definieren wir analog $\mathfrak{p}_{\varphi_{n}, r, s}$ als das von

$$
\begin{aligned}
& g_{\varphi_{n}, r, s}=g_{\varphi_{n}, r}+A_{n+2} q_{\varphi_{n}, r, s}^{r_{\varphi_{n}, r, s}} \\
& h_{\varphi_{n}, r, s}=h_{\varphi_{n}, r}+B_{n+2} q_{\varphi_{n}, r, s}^{r_{\varphi_{n}, r, s}}
\end{aligned}
$$

erzeugte Primideal, wobei $q_{\varphi_{n}, r, s}=q_{\varphi_{n}, r} \cdot \varphi(s)$ und 


$$
r_{\varphi_{n}, r, s}=\left[\left(t_{\varphi_{n}, r, s}+1\right) !\right]^{2} \text { mit } t_{\varphi_{n}, r, s}=\partial q_{\varphi_{n}, r, s} .
$$

Bemerkung. Da für alle $k \in N, \mathfrak{p}_{\varphi_{n}}, X+Y^{k}$ und $Y$ das maximale Ideal in $K[X, Y, Z, T]_{(X, Y, Z, T)}$ erzeugen, gibt es unendlich viele Primelemente in $\mathscr{P}$, die nicht in $\mathfrak{p}_{\varphi_{n}}$ liegen.

SAtz 2. Es gibt eine Abzählung $\psi: N \rightarrow \mathscr{P}$ mit $\psi(1)=X$ und $\psi(n)$ $\notin \mathfrak{p}_{\psi_{n-1}}$ für alle $n \in N$.

Zum Beweis benötigen wir den folgenden

Hilfssatz. $\mu: N \rightarrow \mathscr{P}$ sei eine Abzählung von $\mathscr{P}$ mit $\mu(1)=X$ und $\mu(i)$ $\notin \mathfrak{p}_{\mu_{i-1}}$ für alle $i \leqq n$. Ist $\mu(n+1) \in \mathfrak{p}_{\mu_{n}}$, so ist eine der folgenden Bedingungen erfüllt:

(i) Es gibt ein $t>n+1$ mit

$$
\mu(t) \notin \mathfrak{p}_{\mu_{n}}, \quad \mu(n+1) \notin \mathfrak{p}_{\mu_{n, t}}
$$

(ii) Es gibt $m, t \in N, m>t>n+1 m i t$

$$
\mu(t) \notin \mathfrak{p}_{\mu_{n}}, \quad \mu(m) \notin \mathfrak{p}_{\mu_{n, \ell}}, \quad \mu(n+1) \notin \mathfrak{p}_{\mu_{n, t, m}} .
$$

Beweis des Hilfssatzes.

1. Fall. Es gibt ein $i>n+1$ mit $\mu(i) \notin \mathfrak{p}_{\mu_{n}}$ und $\mu(n+1) \notin \mathfrak{p}_{\mu_{n}, i}$. Dann sind wir fertig.

2. Fall. Für alle $i>n+1$ mit $\mu(i) \notin \mathfrak{p}_{\mu_{n}}$ folgt: $p=\mu(n+1) \in \mathfrak{p}_{\mu_{n}, i}$. Die Menge dieser $i \in N$ werde mit $I_{0}$ bezeichnet. Offenbar enthält $I_{0}$ unendlich viele Elemente. Wegen $p \in \mathfrak{p}_{\mu_{n}}$ gibt es also $\alpha, \beta, \alpha_{i}, \beta_{i} \in K[X, Y, Z, T]_{(X, Y, Z, T)}$ mit: $p=\alpha g_{\mu_{n}}+\beta h_{\mu_{n}}=\alpha_{i} g_{\mu_{n}, i}+\beta_{i} h_{\mu_{n}, i}$ für alle $i \in I_{0}$. Subtraktion liefert:

$$
0=\left(\alpha-\alpha_{i}\right) g_{\mu_{n}, i}+\left(\beta-\beta_{i}\right) h_{\mu_{n}, i}-\left(\alpha A_{n+1}+\beta B_{n+1}\right) q_{\mu_{n}, i}^{\mu_{n}, i}
$$

Damit folgt:

$q_{\mu_{n}, i}^{r_{n}, i}\left(\alpha A_{n+1}+\beta B_{n+1}\right) \in \mathfrak{p}_{\mu_{n}, i}$ für alle $i \in I_{0}$. Wäre $q_{\mu_{n}, i} \in \mathfrak{p}_{\mu_{n}, i}$, so folgt $\mu(i) \in \mathfrak{p}_{\mu_{n}, i}$ oder $\mu(j) \in \mathfrak{p}_{\mu_{n}, i}$ für ein $j \leqq n$. Daraus ergibt sich $\mathfrak{p}_{\mu_{n, i}}=\mathfrak{p}_{\mu_{n}}$ bzw. $\mathfrak{p}_{\mu_{j-1}}=\mathfrak{p}_{\mu_{n}, i}$ und damit der Widerspruch $\mu(i) \in \mathfrak{p}_{\mu_{n}}$ bzw. $\mu(j) \in \mathfrak{p}_{\mu_{j-1}}$ entgegen der Voraussetzung. Also folgt für alle $i \in I_{0}$ :

$$
\alpha A_{n+1}+\beta B_{n+1} \in \mathfrak{p}_{\mu_{n}, i}
$$

Insbesondere folgt:

$$
\mu(n+1)=p, \quad \alpha A_{n+1}+\beta B_{n+1} \in \bigcap_{i \in I_{0}} \mathfrak{p}_{\mu_{n}, i}
$$


Behauptung (*). Es gibt eine Teilmenge $J \subseteq I_{0}$ mit $|J|=\infty$ und folgender Eigenschaft: Für alle $i, j \in J$ mit $i \neq j$ ist

$$
\mathfrak{p}_{\mu_{n}, i} \neq \mathfrak{p}_{\mu_{n}, j} \text {. }
$$

Beweis von (*). Für alle $k \in N$ ist $X+Y^{k} \notin \mathfrak{p}_{\mu_{n}}$, also gibt es ein $k_{0}$ $\in N$ mit folgender Eigenschaft: $\mathrm{Zu}$ jedem $k \geqq k_{0}$ gibt es ein $i_{k} \in I_{0}$ mit $\mu\left(i_{k}\right)=X+Y^{k}$.

Für $k>\ell \geqq k_{0}$ ist aber $\mathfrak{p}_{\mu_{n}, i_{k}} \neq \mathfrak{p}_{\mu_{n}, i_{\ell}}$, da andernfalls

$$
\begin{aligned}
& g_{\mu_{n}, i_{k}}-g_{\mu_{n}, i_{\ell}} \\
& =A_{n+\ell} q_{\mu_{n}}^{r_{n}, i_{\ell}}\left[q_{\mu_{n}}^{\left(r_{\mu_{n}, i_{k}}-r_{\left.\mu_{n}, i_{\ell}\right)}\right.} \mu\left(i_{k}\right)^{r_{n}, i_{k}}-\mu\left(i_{\ell}\right)^{r_{\mu_{n}, i_{\ell}}}\right] \\
& \in \mathfrak{p}_{\mu_{n}, i_{k}}=\mathfrak{p}_{\mu_{n, i}} \\
& \rightarrow q_{\mu_{n}}^{t} \mu\left(i_{k}\right)^{v}-\mu\left(i_{\ell}\right)^{u} \in \mathfrak{p}_{\mu_{n}, i_{k}}=\mathfrak{p}_{\mu_{n}, i_{\ell}}
\end{aligned}
$$

wobei $t=r_{\mu_{n}, i_{k}}-r_{\mu_{n}, i_{\ell}} ; v=r_{\mu_{n}, i_{k}} ; u=r_{\mu_{n}, i_{\ell}}$ und $v>u$, wegen $k>1$. Dann ist aber

$$
q_{\mu_{n}}^{t} X^{v}-X^{u} \in\left(\mathfrak{p}_{\mu_{n}, i_{k}}, Y\right) \in \operatorname{Spec}\left(K[X, Y, Z, T]_{(X, Y, Z, T)},\right.
$$

und damit $X \in\left(\mathfrak{p}_{\mu_{n}, i_{k}} ; Y\right)$ im Widerspruch $z u(X, Y, Z, T)=\left(\mathfrak{p}_{\mu_{n}, i_{k}}, X, Y\right)$ und Höhe $\mathfrak{p}_{\mu_{n}, i_{k}}=2$. Damit ist die Behauptung $(*)$ bewiesen.

Aus (*) folgt nun Höhe $\left(\bigcap_{i \in I} \mathfrak{p}_{\mu_{n}, i}\right)=1$ und wegen $p \in \bigcap_{i \in I} \mathfrak{p}_{\mu_{n}, i}, p$ Primelement ergibt sich:

$$
\bigcap_{i \in I} \mathfrak{p}_{\mu_{n}, i}=(p)
$$

Daraus folgt: $p$ teilt $\alpha A_{n+1}+\beta B_{n+1}$ in $K[X, Y, Z, T]_{(X, Y, Z, T)}$. Sei nun $i_{0} \in I_{0}$ $\subseteq N$ minimal.

Fall 2.1. Es gibt ein $m>i_{0}$ mit $\mu(m) \notin \mathfrak{p}_{\mu_{n}, i_{0}}$ und $p \notin \mathfrak{p}_{\mu_{n}, i_{0}, m}$. Dann ist nichts mehr zu zeigen.

Fall 2.2. Für alle $i>i_{0}$ mit $\mu(i) \notin \mathfrak{p}_{\mu_{n}, i_{0}}$ ist $p \in \mathfrak{p}_{\mu_{n}, i_{0}, i}$. Dann gibt es wieder $\alpha_{i}, \beta_{i} \in K[X, Y, Z, T]_{(X, Y, Z, T)}$ mit $p=\alpha g_{\mu_{n}}+\beta h_{\mu_{n}}=\alpha_{i} g_{\mu_{n}, i_{0}, i}+\beta_{i} h_{\mu_{n}, i_{0}, i}$. Durch Subtraktion erhält man daraus:

$$
\begin{aligned}
0= & \left(\alpha-\alpha_{i}\right) g_{\mu_{n}, i_{0}, i}+\left(\beta-\beta_{i}\right) h_{\mu_{n}, i_{0}, i}-\alpha\left(A_{n+1} q_{\mu_{n}, i_{0}}^{r_{\mu_{n}, i_{0}}}+A_{n+2} q_{\mu_{n}, i_{0}, i}^{r_{\mu_{n}, i_{0}, i}}\right) \\
& -\beta\left(B_{n+1} q_{\mu_{n}, i_{0}}^{r_{\mu_{n}, i_{0}}}+B_{n+2} q_{\mu_{n}, i_{0}, i}^{r_{\mu_{n}, i}, i}\right)
\end{aligned}
$$

Wir setzen:

$$
\begin{aligned}
F= & \alpha\left(A_{n+1} q_{\mu_{n}, i_{0}}^{r_{\mu_{n}, i_{0}}}+A_{n+2} q_{\mu_{n}, i_{0}, i}^{r_{\mu_{n}, i_{0}, i}}\right) \\
& +\beta\left(B_{n+1} q_{\mu_{n}, i_{0}}^{r_{\mu_{n}, i_{0}}}+B_{n+2} q_{\mu_{n}, i_{0}, i}^{r_{\mu_{n}, i_{0}, i}}\right)
\end{aligned}
$$


Da $\alpha A_{n+1}+\beta B_{n+1} \in(p)$ und da $p \in \mathfrak{p}_{\mu_{n}, i_{0}, i}$ für alle $i$, folgt:

$$
G=\left(\alpha A_{n+2}+\beta B_{n+2}\right) q_{\mu_{n}, i_{0}, i}^{r_{\mu_{n}, i_{0}, i} \in \mathfrak{p}_{\mu_{n}, i_{0}, i}}
$$

für alle $i$ mit $i>i_{0}$ und $\mu(i) \notin \mathfrak{p}_{\mu_{n}, i_{0}}$. Daraus folgt wieder $\alpha A_{n+2}+\beta B_{n+2}$ $\in \mathfrak{p}_{\mu_{n}, i_{0}, i}$ für alle $i>i_{0}$ mit $\mu(i) \notin \mathfrak{p}_{\mu_{n}, i_{0}, i}$ und ebenso wie oben ergibt sich dann: $p$ teilt $\alpha A_{n+2}+\beta B_{n+2}$. Insgesamt erhalten wir also: $p$ teilt $\alpha A_{n+1}$ $+\beta B_{n+1}$ und $p$ teilt $\alpha A_{n+2}+\beta B_{n+2}$. Daraus folgt: $p$ teilt $\beta\left(B_{n+1} A_{n+2}\right.$ - $\left.B_{n+2} A_{n+1}\right)$ und da $B_{n+1} A_{n+2}-A_{n+1} B_{n+2}$ eine Einheit in $K$ ist, folgt: $p$ teilt $\alpha$ und $p$ teilt $\beta$, d.h. $\alpha=p \cdot \gamma$ und $\beta=p \cdot \delta$ mit $\gamma, \delta \in K[X, Y, Z$, $T]_{(X, Y, Z, T)}$. Daraus ergibt sich dann der Widerspruch $p=p\left(\gamma g_{\mu_{n}}+\beta h_{\mu_{n}}\right)$, da $\gamma g_{\mu_{n}}+\beta h_{\mu_{n}} \in \mathfrak{p}_{\mu_{n}}$ keine Einheit in $K[X, Y, Z, T]_{(X, Y, Z, T)}$ ist. Damit ist der Hilfssatz bewiesen.

Beweis von Satz 2. $\varphi: N \rightarrow \mathscr{P}$ sei eine Abzählung von $\mathscr{P}$ mit $\varphi(1)=X$. Wir definieren $\Psi: N \rightarrow \mathscr{P}$ rekursiv. Sei $\Psi(1)=\varphi(1)=X$ und für $i \leqq n$ sei $\Psi(i)$ bereits definiert mit $\Psi(i) \notin \mathfrak{p}_{\Psi_{i-1}}$ für alle $2 \leqq i \leqq n$. Sei $k \in N$ minimal mit $\varphi(k) \in \mathscr{P} \backslash\{\Psi(1), \cdots, \Psi(n)\}$.

1. Fall. $\varphi(k) \notin \mathfrak{p}_{\psi_{n}}$

Definiere $\Psi(n+1):=\varphi(k)$

2. Fall. $\varphi(k) \in \mathfrak{p}_{\Psi_{n}}$

Dann gibt es eine Abzählung $\mu: N \rightarrow \mathscr{P}$ mit

$$
\begin{aligned}
\mu(i) & =\Psi(i) \quad \text { für alle } i \leqq n \\
\mu(n+1) & =\varphi(k)
\end{aligned}
$$

und

$$
\mu(r)=\varphi(r) \quad \text { für alle } r>r_{0}>n
$$

wobei $r_{0} \in N$ hinreichend groß zu wählen ist. Nach dem Hilfssat's gibt es natürliche Zahlen $m \geqslant t>n+1$ mit: $\mu(t) \notin \mathfrak{p}_{\mu_{n}}=\mathfrak{p}_{\Psi_{n}}, \mu(m) \notin \mathfrak{p}_{\mu_{n}, t}$ und $\mu(n+1) \notin \mathfrak{p}_{\mu_{n}, t}$ oder $\mu(n+1) \notin \mathfrak{p}_{\mu_{n}, t, m}$. Falls $\mu(n+1) \notin \mathfrak{p}_{\mu_{n}, t}$ ist, so setze $\operatorname{man} \Psi(n+1):=\mu(t)$ und $\Psi(n+2):=\varphi(k)=\mu(n+1)$. Ist $\mu(n+1) \in \mathfrak{p}_{\mu_{n}, t}$ und $\mu(n+1) \notin \mathfrak{p}_{\mu_{n}, t, m}$, so sei $\Psi(n+1):=\mu(t) ; \Psi(n+2):=\mu(m)$ und $\Psi(n$ $+3)=\varphi(k)=\mu(n+1)$. Damit folgt Satz 2.

\section{§2. Konstruktion}

Nach Satz 2 gibt es eine Abzählung $\mathscr{P}=\left\{p_{n}\right\}_{n \in N}$ von $\mathscr{P}$ mit $p_{1}=X$ und $p_{n+1} \notin \mathfrak{p}_{n}$ für alle $n \in N$, wobei $\mathfrak{p}_{n}=\left(g_{n}, h_{n}\right)$ mit 


$$
\begin{aligned}
& g_{n}=Z+\sum_{i=1}^{n} A_{i} q_{i}^{r_{i}} ; h_{n}=T+\sum_{i=1}^{n} B_{i} q_{i}^{r_{i}} ; \\
& q_{k}=\prod_{i=1}^{k} p_{i} ; r_{k}=\left[\left(t_{k}+1\right) !\right]^{2}, \quad t_{k}=\partial q_{k} .
\end{aligned}
$$

Eine solche Abzählung von $\mathscr{P}$ sei im folgenden fest gewählt. Wir bilden nun folgende Elemente:

$$
\omega_{0}=\left(Z+\sum_{i=1}^{\infty} A_{i} q_{i}^{r_{i}}\right)\left(T+\sum_{i=1}^{\infty} B_{i} q_{i}^{r_{i}}\right) \in K[[X, Y, Z, T]]
$$

und für $n \geqq 1$ :

$$
\begin{aligned}
\omega_{n}= & \frac{\omega_{0}-g_{n} h_{n}}{q_{n}^{r_{n}}} \\
= & \frac{1}{q_{n}^{r_{n}}}\left[\left(g_{n}+\sum_{i=n+1}^{\infty} A_{i} q_{i}^{r_{i}}\right)\left(h_{n}+\sum_{i=n+1}^{\infty} B_{i} q_{i}^{r_{i}}\right)-g_{n} h_{n}\right] \\
= & \frac{1}{q_{n}^{r_{n}}}\left[g_{n} \sum_{i=n+1}^{\infty} B_{i} q_{i}^{r_{i}}+h_{n} \sum_{i=n+1}^{\infty} A_{i} q_{i}^{r_{i}}\right. \\
& \left.+\sum_{i=n+1}^{\infty} A_{i} q_{i}^{r_{i}} \sum_{i=n+1}^{\infty} B_{i} q_{i}^{r_{i}}\right]
\end{aligned}
$$

Da $q_{n}^{r_{n}} q_{n+k}^{r_{n}+k}$ für alle $n, k \in N$ in $K[X, Y, Z, T]$ teilt, ist $\omega_{n} \in K[[X, Y, Z, T]]$ für alle $n \in N$. Zwischen $\omega_{n}$ und $\omega_{n+1}$ besteht für alle $n \in N$ folgender Zusammenhang:

$$
\omega_{0}=q_{1}^{r_{1}} \omega_{1}+g_{1} h_{1}
$$

(2a) und für $n \in N$

$$
\omega_{n}=\frac{q_{n+1}^{r_{n+1}}}{q_{n}^{r_{n}}}\left(\omega_{n+1}+A_{n+1} h_{n}+B_{n+1} g_{n}+A_{n+1} B_{n+1} q_{n+1}^{r_{n+1}}\right)
$$

wobei $q_{1}, q_{n+1}^{r_{n+1}} / q_{n}^{r_{n}} \in(X, Y, Z, T) K[X, Y, Z, T]$. Wir bilden nun in $K[[X$, $Y, Z, T]]$ den Unterring

$$
R^{1}=K\left[X, Y, Z, T, \omega_{n}\right]_{n \in N}
$$

Aus der Rekursionsformel (2a) folgt, daß $X, Y, Z, T$ ein maximales Ideal in $R^{1}$ erzeugen. Wir setzen:

$$
R=R_{(X, Y, Z, T)}^{1}
$$

Dann folgt:

(2.1) Die kanonischen Einbettungsmorphismen 


$$
K[X, Y, Z, T]_{(X, Y, Z, T)} \rightarrow R \rightarrow K[[X, Y, Z, T]]
$$

sind lokal, das maximale Ideal in $K[X, Y, Z, T]_{(X, Y, Z, T)}$ erzeugt das maximale Ideal von $R$.

(2.2) Ist $\left.S=K[X, Y, Z, T]_{(X, Y, Z, T)}\right)\{0\}$, dann ist $S^{-1} R$ isomorph zu einem Quotientenring von $K(X, Y, Z, T)\left[\omega_{0}\right]$; insbesondere ist $S^{-1} R$ ein noetherscher Ring der Dimension 1. Im folgenden wollen wir zeigen, daß $A=R /\left(\omega_{0}\right)$ ein lokaler universell japanischer Ring ist, dessen regulärer Ort Reg $(A)$ nicht offen in Spec $(A)$ ist. Ferner folgt, daß $A$ Restklassernring eines regulären lokalen Ringes der Dimension 4 ist, der einen Körper der Charakteristik 0 umfaßt.

\section{§3. Noetherzität von $\mathbf{R}$}

Für alle $n \in N_{0}$ ist der Unterring $K\left[X, Y, Z, T, \omega_{n}\right]$ von $K[[X, Y, Z, T]]$ freie $K$-Algebra der Dimension 5. Wir setzen $R_{n}=K\left[X, Y, Z, T, \omega_{n}\right]_{m_{n}} \subseteq R$, wobei $m_{n}$ das von $X, Y, Z, T$ und $\omega_{n}$ erzeugte maximale Ideal in $K[X, Y$, $\left.Z, T, \omega_{n}\right]$ ist. $R_{n}$ ist ein regulärer lokaler Ring der Dimension 5 und enthalten in $R$. Der lokale Einbettungsmorphismus $R_{n} \stackrel{\nu_{n}}{\longrightarrow} R$ faktorisiert für alle $n \in N$ über:

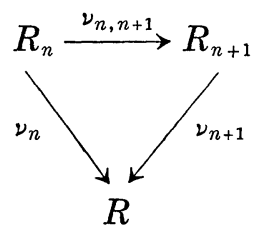

wobei $\nu_{n, n+1}$ induziert wird von dem $K$-Algebramorphismus:

$$
\begin{aligned}
& \bar{\nu}_{n, n+1}: K\left[X, Y, Z, T, \omega_{n}\right] \longrightarrow K\left[X, Y, Z, T, \omega_{n+1}\right] \\
& K[X, Y, Z, T] \stackrel{\text { id }}{\longrightarrow} K[X, Y, Z, T] \\
& \omega_{n} \longmapsto \begin{cases}\frac{q_{n+1}^{r_{n}+1}}{q_{n}^{r_{n}}}\left[\omega_{n+1}+A_{n+1} h_{n}+B_{n+1} g_{n}+A_{n+1} B_{n+1} q_{n+1}^{r_{n+1}}\right] \\
q_{n} \quad \text { falls } n \geqq 1 \\
q_{1}^{r_{1} \omega_{1}}+q_{1} h_{1} \quad \text { falls } n=0\end{cases}
\end{aligned}
$$

Für $n<m$ definieren wir $\nu_{n, m}$ als den zusammengesetzten Morphismus $\nu_{m+1, m} \circ \cdots \circ \nu_{n, n+1}$. Damit läßt sich $R$ auffassen als direkter Limes über $\left(R_{n}, \nu_{n, m}\right)_{n, m \in N}$. 
SATz 3. $R$ ist ein noetherscher Ring.

Beweis. $\mathrm{Zu}$ zeigen ist, daß alle Primideale von $R$ endlich erzeugt sind. Sei also $\mathfrak{p} \in \operatorname{Spec}(R)$.

1. Fall. $\mathfrak{p} \cap K[X, Y, Z, T]_{(X, Y, Z, T)}=\mathfrak{q} \neq(0)$. Dann ist $R / \mathfrak{q} R$ Restklassenring von $K[X, Y, Z, T]_{(X, Y, Z, T)}$, also ist $\mathfrak{p}$ endlich erzeugt.

2. Fall. $\mathfrak{p} \cap K[X, Y, Z, T]_{(X, Y, Z, T)}=(0)$ und $\mathfrak{q} \neq(0)$. Mit $\mathfrak{p}_{n}=R_{n} \cap \mathfrak{p}$ ist dann ebenfalls $\mathfrak{p}_{n} \cap K[X, Y, Z, T]=(0)$. Wegen $R_{n}=K\left[X, Y, Z, T, \omega_{n}\right]_{m_{n}}$ folgt: Höhe $\mathfrak{p}_{n}=1$ für alle $n \in N$ : also ist auch Höhe $\mathfrak{p}=1$. Dann gibt es für alle $n \in N$ ein $P_{n} \in R_{n}$ mit $\left(P_{n}\right)=\mathfrak{p}_{n}$, da $R_{n}$ ein faktorieller Ring ist. Ferner gilt: $\nu_{n, n+1}\left(P_{n}\right) \in \mathfrak{p}_{n+1}$, also $\nu_{n, n+1}\left(P_{n}\right)=\alpha_{n, n+1} P_{n+1}$, wobei $\alpha_{n, n+1}$ $\in R_{n+1}$. Der kanonische Einbettungsmorphismus $R_{n} \rightarrow K[[X, Y, Z, T]]$ ist lokal und faktorisiert über $\nu_{n, n+1}: R_{n} \rightarrow R_{n+1}$, und $P_{n}$ zerfällt in $K[[X, Y, Z, T]]$ in ein endliches Produkt irreduzibler Faktoren. Damit folgt: es gibt ein $n_{0} \in N$, so daß $\alpha_{n, n+1}$ für alle $n \geqq n_{0}$ Einheit in $R_{n+1}$ ist. Dann erzeugt $\nu_{n_{0}, n_{0}+r}\left(P_{n_{0}}\right)$ das Ideal $\mathfrak{p}_{n_{0}+r}$ für alle $r \in N$, das bedeutet, daß $P_{n_{0}}$ das Ideal $\mathfrak{p}$ in $R$ erzeugt, und es folgt die Behauptung.

Folgerung. $R$ ist ein regulärer lokaler Ring der Dimension 4. Da die Einbettungsmorphismen $K[X, Y, Z, T]_{(X, Y, Z, T)} \rightarrow R, R \rightarrow K[[X, Y, Z, T]]$ lokal sind und da $X, Y, Z, T$ das maximale Ideal von $R$ erzeugen, folgt: $\hat{R} \cong K[[X, Y, Z, T]]$.

Lemma. $\omega_{0}$ ist ein Primelement in $R$.

Beweis. $\omega_{0}$ ist Primelement oder Einheit in $S^{-1} R$, da $S^{-1} R$ ein Quotientenring von $K\left[X, Y, Z, T, \omega_{0}\right]$ ist. Es genügt nachzuweisen, daß $\omega_{0} \notin\left(p_{n}\right)$ für alle $n \in N$. Annahme: $p_{n}$ teilt $\omega_{0}=\left(g_{n-1}+p_{n} \tilde{g}_{n}\right)\left(h_{n-1}+p_{n} \tilde{h}_{n-1}\right)$ mit $\tilde{g}, \tilde{h}_{n} \in K[[X, Y, Z, T]]$. Dann ist $g_{n-1}$ oder $h_{n-1}$ durch $p_{n}$ teilbar. Da $g_{n-1}$ und $h_{n-1}$ Primelemente sind, folgt, daß $p_{n} \mathrm{zu} g_{n-1}$ oder zu $h_{n-1}$ assoziiert ist, im Widerspruch zu $p_{n} \notin \mathfrak{p}_{n-1}$.

\section{§4. A ist ein lokaler universell japanischer Ring mit nicht offenem regulärem Ort}

SATz 4. A ist universell japanisch.

Beweis. Da $Q \subseteq A$ ist nach Theorem 1 (iii) nur zu zeigen: Für alle $\mathfrak{p} \in \operatorname{Spec}(A)$ ist $\mathfrak{p} \hat{A}$ reduziert. Sei also $\mathfrak{p} \in \operatorname{Spec}(A)$

1. Fall $\mathfrak{p}=(0)$. 


$$
\hat{A}=\widehat{R /\left(\omega_{0}\right)}=K[[X, Y, Z, T]] / \omega_{0} K[[X, Y, Z, T]]
$$

ist reduziert, da $\omega_{0}$ in $K[[X, Y, Z, T]]$ Produkt zweier nicht assoziierter Primelemente ist, d.h.: $\omega_{0}=U . V$, wobei $U=Z+\sum_{n=1}^{\infty} A_{n} q_{n}^{r_{n}} ; V=T$ $+\sum_{n=1}^{\infty} B_{n} q_{n}^{r_{n}}$. $U$ und $V$ sind nicht assoziiert, da $U, V, X$ und $Y$ das maximale Ideal von $K[[X, Y, Z, T]]$ erzeugen.

2. Fall. $\mathfrak{p} \neq(0) . \quad \mu: R \rightarrow R /\left(\omega_{0}\right)$ sei der kanonische Restklassenmorphismus. Dann gibt es ein $\mathfrak{\beta} \in \operatorname{Spec}(R)$ mit $\mathfrak{R} \supseteq\left(\omega_{0}\right)$ und $\mu(\mathfrak{P})=\mathfrak{p}$. Wegen Höhe $\mathfrak{p} \geqq 1$ ist Höhe $\mathfrak{\beta} \geqq 2$; also folgt: $\mathfrak{\beta} \cap K[X, Y, Z, T] \neq(0)$; d.h. es gibt ein $n \in N$ mit $p_{n} \in \mathfrak{P}$. Dann ist $R / \mathfrak{P}=A / \mathfrak{p}$ isomorph zu einem Restklassenring von $K[X, Y, Z, T]_{(X, Y, Z, T)}$ und es folgt; $\widehat{(A / \mathfrak{P})}$ ist reduziert. Damit ist Satz 4 bewiesen. Im folgenden sei: $U:=Z+\sum_{n=1}^{\infty} A_{n} q_{n}^{r_{n}}$ und $V:=T+\sum_{n=1}^{\infty} B_{n} q_{n}^{r_{n}}$. Dann folgt:

Lemma 1. $(U, V)$ ist ein Primideal in $K[[X, Y, Z, T]]$ mit $(U, V) \cap R$ $=\left(\omega_{0}\right)$.

Beweis. $U$ und $V$ sind Teil eines regulären Parametersystems, also ist $(U, V)$ Primideal in $K[[X, Y, Z, T]]$. Ferner ist $(U, V) \cap K[X, Y, Z, T]$ $=(0)$, denn andernfalls gibt es ein $p_{n} \in \mathscr{P}$ mit $p_{n} \in(U, V)$; dann folgt: $g_{n-1}, h_{n-1} \in(U, V)$. Da $\left(g_{n-1}, h_{n-1}\right)$ ebenfalls in $K[[X, Y, Z, T]]$ ein Primideal der Höhe 2 ist, folgt $\left(g_{n-1}, h_{n-1}\right)=(U, V)$ und daraus $p_{n} \in \mathfrak{p}_{n-1}$ in $K[X, Y$, $Z, T]_{(X, Y, Z, T)}$ im Widerspruch zur Konstruktion. Also ist Höhe $(U, V)$ $\cap R=1$ und wegen $\omega_{0} \in R \cap(U, V), \omega_{0}$ Primelement in $R$, folgt: $(U, V)$ $\cap R=\left(\omega_{0}\right)$.

Für alle $n \in N$ gilt: $\left(\mathfrak{p}_{n-1}, p_{n}\right) K[[X, Y, Z, T]] \supseteq(U, V)$. Nun gibt es für alle $i \in N$ ein $n_{i} \in N$ mit $p_{n_{i}}=X+Y^{i}$ und $\left.\mathfrak{q}_{i}=\left(p_{n_{i}}, p_{n_{i}}\right) \in \operatorname{Spec}(K[X, Y, Z, T])_{(X, Y, Z, T)}\right)$; ferner ist $\mathfrak{q}_{i} R \in \operatorname{Spec}(R)$ und $\mathfrak{q}_{i} K[[X, Y, Z, T]] \in \operatorname{Spec}(K[[X, Y, Z, T]])$

Lemma 2. Für alle $i, j \in N$ mit $i \neq j$ ist $\mathfrak{q}_{i} \neq \mathfrak{q}_{j}$.

Beweis: Annahme: Es gibt $i, j \in N$ mit $\mathfrak{q}_{i}=\mathfrak{q}_{j}$ und $i \neq j$. Sei etwa $i>j$. Dann gibt es $\alpha, \beta, \gamma \in K[X, Y, Z, T]_{(X, Y, Z, T)}$ mit $p_{n_{j}}=\alpha g_{n_{i}}+\beta h_{n_{i}}+\gamma p_{n_{i}}$, $\alpha, \beta, \gamma$ lassen sich darstellen als $\alpha=a / e, \beta=b / e$ und $\gamma=c / e$ mit $a, b, c, e$ $\in K[X, Y, Z, T] ; e \notin(X, Y, Z, T)$. Damit folgt:

$$
\left(X+Y^{j}\right) e=a g_{n_{i}}+b h_{n_{i}}+c\left(X+Y^{i}\right)
$$

Auf der linken Seite tritt ein Term $Y^{j} e_{0} ; e_{0} \in K \backslash\{0\}$ auf, auf der rechten Seite enthalten $a g_{n_{i}}$ bzw. $b h_{n_{i}}$ nur Terme $Y^{k} f$, wobei $f \in(X, Z, T)$ ist, in 
$c\left(X+Y^{i}\right)$ tritt ebenfalls kein Term der Form $e_{0} Y^{j}$ auf, wegen $i>j$. Also ist die Annahme falsch und es folgt: $\mathfrak{q}_{i} \neq \mathfrak{q}_{j}$.

Satz 5. Sing $(A)$ ist nicht abgeschlossen in Spes $(A)$.

Beweis. $\quad q$ sei das von $U$ und $V$ in $\hat{A}=K[[X, Y, Z, T]] / \omega_{0} K[[X, Y, Z, T]]$ erzeugte Primideal. Für alle $\mathfrak{\beta} \in V(\mathfrak{q})$ ist $\hat{A}_{\mathfrak{B}}$ nicht integer, also nicht regulär, damit ist $V(\mathfrak{q}) \subseteq \operatorname{Sing}(\hat{A})$. Ferner ist für die $\mathfrak{q}_{i}$ aus Lemma 2: $\mathfrak{q}_{i} \hat{A} \in V(\mathfrak{q})$ für alle $i \in N$. Mit dem Lemma nach Theorem 1 folgt dann: $\mathfrak{q}_{i} A \in \operatorname{Sing}(A)$ für alle $i \in N$. Da $A$ Integritätsbereich ist, genügt es nun zu zeigen: $\bigcap_{i \in N} \mathfrak{q}_{i} A=(0)$. Dazu brauchen wir nur nachzuweisen, daß $\bigcap_{i \in N} \mathfrak{q}_{i} R=\omega_{0} R$ ist. Nun gilt: $\bigcap_{i \in N} \mathfrak{q}_{i} R \cong\left(\bigcap_{i \in N} \mathfrak{q}_{i} \hat{R}\right) \cap R$ und $\bigcap_{i \in N} \mathfrak{q}_{i} \hat{R} \supseteq(U, V)$. Die $\mathfrak{q}_{i} \hat{R}$ sind paarweise verschiedene Primideale der Höhe 3 , dann ist $\bigcap_{i \in N} \mathfrak{q}_{i} \hat{R}$ ein Ideal der Höhe 2, das ein Primideal der Höhe 2 umfaßt, nämlich $(U, V)$. Also ist $\bigcap_{i \in N} \mathfrak{q}_{i} \hat{R}=(U, V)$ und mit Lemma 1 folgt: $\bigcap_{i \in N} \mathfrak{q}_{i} R$ $=\omega_{0} R$.

\section{LITERATUR}

[1] Bourbaki, N., Commutative Algebra, Paris, Hermann 1972.

[2] Grothendieck, A., Élements de Geométrie algebrique, Inst. haut. Etud. sci., Pub]. math. 24 (1965).

[ 3 ] Marot, J.: Sur les anneaux universellement japonais, Bull. Soc. math. France, 103 (1975), 103-111.

[4] Matsumura, H., Formal power series rings over polynomial rings I, in Number Theory, Algebraic Geometry and Commutative Algebra in honour of Y. Akizuki. Tokyo, Kinokuniya 1973.

[5] Nagata, M., Local Rings, Interscience Tracts in Pure and Applied Mathematics Nr. 13, New York, Interscience 1962.

[ 6 ] Nomura, M., Formal power series rings over polynomial rings II, Number Theory, Algebraic Geometry and Commutative Algebra in honour of Y. Akizuki, Tokyo, Kinokuniya (1973).

[7] Rotthaus, C., Nicht ausgezeichnete, universell japanische Ringe, Math. Z. 152 (1977), 107-125.

[8] Valabrega, P, A few theorems on Completion of excellent rings, Nagoya Math. J., Vol. 61 (1976), 127-133.

\section{Universität Münster}

